\title{
E-selectin ligand-1 controls circulating prostate cancer cell rolling/adhesion and metastasis
}

\author{
Sayeda Yasmin-Karim ${ }^{1}$, Michael R. King ${ }^{2}$, Edward M. Messing ${ }^{1}$, Yi-Fen Lee ${ }^{1}$ \\ ${ }^{1}$ Departments of Urology and Pathology and Laboratory Medicine, and Chemical Engineering, University of Rochester, \\ Rochester, NY 14642 \\ ${ }^{2}$ Department of Biomedical Engineering, Cornell University, Ithaca, NY 14853 \\ Correspondence to: \\ Yi-Fen Lee, e-mail: YiFen_Lee@URMC.Rochester.edu
}

Keywords: prostate cancer, circulatory tumor cell, cancer cell rolling and adhesion, E-selectin ligand-1, E-selectin

Received: July 31, $2014 \quad$ Accepted: September 16, $2014 \quad$ Published: October 06, 2014

\section{ABSTRACT}

Circulating prostate cancer ( $\mathrm{PCa}$ ) cells preferentially roll and adhere on bone marrow vascular endothelial cells, where abundant E-selectin and stromal cell-derived factor 1 (SDF-1) are expressed, subsequently initiating a cascade of activation events that eventually lead to the development of metastases. To elucidate the roles of circulating PCa cells' rolling and adhesion behaviors in cancer metastases, we applied a dynamic cylindrical flow-based microchannel device that is coated with E-selectin and SDF-1, mimicking capillary endothelium. Using this device we captured a small fraction of rolling PCa cells. These rolling cells display higher static adhesion ability, more aggressive cancer phenotypes and stem-like properties. Importantly, mice received rolling PCa cells, but not floating PCa cells, developed cancer metastases. Genes coding for E-selectin ligands and genes associated with cancer stem cells and metastasis were elevated in rolling PCa cells. Knock down of E-selectin ligand 1(ESL-1), significantly impaired PCa cells' rolling capacity and reduced cancer aggressiveness. Moreover, ESL-1 activates RAS and MAP kinase signal cascade, consequently inducing the downstream targets. In summary, circulating PCa cells' rolling capacity contributes to PCa metastasis, and that is in part controlled by ESL-1.

\section{INTRODUCTION}

Metastasis is the most dreaded stage of cancer, accounting for the vast majority of prostate cancer (PCa)related deaths [1-3]. Identifying the population at risk for developing advanced $\mathrm{PCa}$ and preventing metastases is one of the greatest challenges in cancer treatment today. Cancer metastases are the culmination of a complex series of steps where cancer cells degrade extracellular matrices allowing them to break away from the primary tumors. During this process, a subset of primary tumor cells undergo epithelial-mesenchymal transformation (EMT), involving morphogenetic changes to a mesenchymal cell-like phenotype of enhanced motility and plasticity that allows them to intravasate into the circulation and become circulating tumor cells (CTCs) [4-6]. Some CTCs can survive in the circulation, and eventually extravasate into distant metastatic locations [3], thus CTC numbers in PCa patients have been suggested as disease prognosis biomarker [7].

The process of extravasation involves several steps. First, CTCs roll on the endothelial cell surfaces which express E-selectin. This causes the CTCs to tether and roll in the post capillary venules $[3,8,9]$. This rolling process enables PCa cells to activate integrin signals, which trigger additional adhesion process, resulting in firm adhesions between cancer cells and endothelial cells [10]. This process promotes the transmigration of cancer cells through the endothelium to the metastatic sites $[10,11]$. PCa cells preferentially metastasize to bone through site-specific interactions. Bone marrow endothelial cells express E-selectin, which enables homing of circulating PCa cells expressing E-selectin ligands to bone [12]. Not limited to PCa, the metastatic tumor cells from colon, breast, lung, ovary, and pancreas also have acquired the capacity to roll and adhere to the endothelial 
monolayer through E-selectin [13-16]. Our recent work has shown that alpha-1,3 fucosyltransferase 6 , the key enzyme catalyzing E-selectin ligands, is an important regulator of $\mathrm{PCa}$ metastasis to bone [17]. It is reported that metastatic PCa tumors express elevated surface levels of E-selectin ligands, including CD 44, PSGL-1, ESL1, beta-2-integrins and L-selectin [3, 9, 18-20]. ESL-1, one of E-selectin ligand, is a $\mathrm{Ca}^{2+}$ dependent inducible cell adhesion glycoprotein [21] and is encoded by a single gene locus named Glg1 (Golgi-complex-localized glycoprotein-1), but its roles in cancer metastasis are not well known. In addition to E-selection, the stromal cellderived factor 1 (SDF-1) and its receptor CXCR4 play a critical role in $\mathrm{PCa}$ bone metastasis. The CXCR4 positive PCa cells can form a firm adhesion to the osteocytes in the bone metastatic lesions that secrete/express SDF-1[22].

So far E-selectin has been recognized as the prime adhesion molecule expressed by the endothelium responsible for initiating rolling and adhesion of $\mathrm{PCa}$ cells [8], but there is a scarcity of knowledge about the role of rolling/adhesion of circulating PCa cells in terms of PCa aggressiveness/metastasis and the mechanism behind this. In this report we elucidate the roles of circulating PCa cells rolling and adhesion behavior in the development of metastatic PCa. To create a bone metastatic microenvironment of $\mathrm{PCa}$ we applied a dynamic flowbased E-selectin/SDF-1 coated microchannel system, mimicking bone marrow post capillary venules [23].
We demonstrated that circulating PCa cells' rolling/ adhesion capacity contributes to PCa's distant metastasis, which is mediated via an E-selectin ligand, ESL-1. Consequently, the overexpression of ESL-1 transduces a cascade of signaling facilitating prostate cancer metastasis.

\section{RESULTS}

\section{Circulating PCa cells' rolling capacity contributes to cancer aggressiveness}

To investigate if circulating $\mathrm{PCa}$ cells' rolling/ adhesion behavior is an important $\mathrm{PCa}$ cell characteristic in the development of aggressive disease, we applied a dynamic flow-based system as illustrated in Supplementary Figure S1A and Supplementary Movie 1 [23]. First, we compared the rolling capacity among PCa cell lines with the same origin but different aggressiveness. Two BPH-1 derived cell lines that were originally established from hormone induced BPH-1 maliganant transformation in a tissue recombinant model were chosen [24]. These BPH-1 derived cell lines are $\mathrm{PHEC}_{\mathrm{T}}$ : isolated from primary tumors vs $\mathrm{PHEC}_{\mathrm{M}}$ : isolated from lymph node metastasis. As expected the metastatic PHEC $_{M}$ cells demonstrated more aggressive cancer cellular phenotypes, higher invasiveness (Figure 1A) and higher growth rate (Figure 1B), as compared to the primary PHEC $_{\mathrm{T}}$ cell line. More importantly, PHEC $_{\mathrm{M}}$ displayed higher rolling

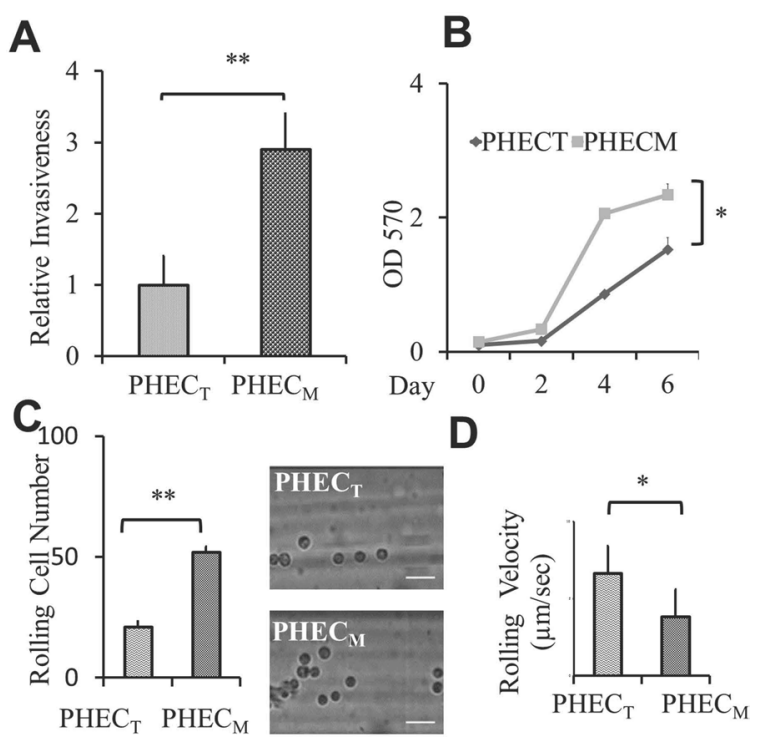

Figure 1: PCa cell aggressiveness is correlated with their rolling/adhesion capacity. (A) Relative invasiveness of BPH1 derived $\mathrm{PHEC}_{\mathrm{T}}$ and $\mathrm{PHEC}_{\mathrm{M}}$ PCa cells. The Bar graph represents invasive cells per well using 24 well invasion chambers, the average of at least three experiments. (B) Line graph represents the MTT assay for cell proliferation. The results represent the average of at least three experiments. (C) Rolling cell number comparing the rolling behavior of BPH-1derived PHEC $_{\mathrm{T}}$ and $\mathrm{PHEC}_{\mathrm{M}}$ cell lines using the microchannel system under wall shear stress of $1 \mathrm{dyne} / \mathrm{cm}^{2}$. Bar graph represents the average rolling/adhesion cell number of consecutive 10 frames of a video (200X) of one experiment. Pictures represent one frame. Scale bar represents $50 \mu \mathrm{m}$. (D) Rolling velocity of BPH1 derived $\mathrm{PHEC}_{\mathrm{T}}$ and $\mathrm{PHEC}_{\mathrm{M}}$ cell lines using the microchannel system under wall shear stress of $1 \mathrm{dyne} / \mathrm{cm}^{2}$. Graph represents the average rolling cell velocity of 10 cells of one experiment. Error bars indicate $\mathrm{SEM}$. *, $\mathrm{P}<0.05$; **, $\mathrm{P}<0.01$. 
cell numbers (Figure 1C) and lower rolling cell velocity (Figure 1D) as compared to primary tumor PHEC $_{\mathrm{T}}$ cells. This positive correlation of cancer aggressiveness with rolling capacity was further confirmed by two pairs of PCa cell lines; LNCaP-P vs. LNCaP-R and CWR22R-1 vs. CWR22R-2 $[25,26]$ where more aggressive PCa cells (Supplementary Figure S1B) also demonstrates higher rolling cell number (Supplementary Figure S1C) and lower rolling cell velocity (Supplementary Figure S1D) compared to their counterpart less aggressive PCa cells. Our data from three sets of PCa cell lines indicated that circulating PCa cells' rolling capacity is correlated with their aggressiveness and $\mathrm{PCa}$ rolling capacity is a novel cancer cell characteristic.

Circulating PCa cells are heterogeneous, and only a small percentage of circulating cancer cells can survive, roll and adhere, and eventually metastasize to a second target site. This flow-based microtube system provides a unique opportunity to isolate and characterize those aggressive circulating PCa cells that roll and adhere to microtubes. Therefore, we used this device to isolate $\mathrm{PCa}$ rolling population from two well-characterized metastatic PCa cell lines, PC-3 and DU145 cells. Cells infused through these coated microtubes under 1 dyne $/ \mathrm{cm}^{2}$ shear stress were sorted and collected according to their adhesion ability (Supplementary Figure S1A). We found that more than $95 \%$ of PC-3 and DU145 cells were floating through the microtubes, defined as "floating" cells, and less than $5 \%$ of PCa cells roll and adhere to the microtubes, defined as "rolling" cells (Supplementary Movie 1). We then characterized these two subpopulations, rolling $v s$ floating $\mathrm{PCa}$ cells, in different in vitro assays for their metastatic potential. First, we examined the sorted $\mathrm{PCa}$ adhesion ability to endothelial cells using the static adhesion assay in which PCa cells are allowed to settle on the endothelium. We found that both PC-3 and DU145 rolling cells have greater adherence to HUVEC cells compared to their corresponding floating cells in the static adhesion assay (Figure 2A and Supplementary Figure S2A). Next, we found that the rolling PC-3 as well as rolling DU145 cells invade more rapidly through the matrigel than corresponding floating cells (Figure 2B and Supplementary Figure S2B), and rolling PCa cells grow faster compare to the corresponding floating cells (Supplementary Figure S2C). Moreover, we performed the soft agar assay to measure the anchorage independent growth, one of the hallmark characteristics of cancer cells. We found that both rolling PC-3 and DU145 cells formed more and bigger colonies than floating $\mathrm{PCa}$ cells (Figure 2C and Supplementary Figure S2D). Finally, the cancer stem cell's characteristic which is associated with cell trafficking [27] and cancer aggressiveness [28] was examined. We compared the stemness between rolling and floating PCa cells by the sphere forming assay, and found rolling $\mathrm{PCa}$ cells developed more and larger sphere colonies in 3-D culture than the floating PCa cells (Figure 2D and Supplementary Figure S2E). Consistent with the cancer stem cell phenotype, we also found that the rolling PC-3 cells express higher levels of stem cell markers, such as NANOG, CD133, CK 5, and prostate specific stem cell marker (PSCA) $[28,29]$ compared to floating PC-3 cells by QPCR (Figure 2E). In summary, we successfully captured a small population of rolling $\mathrm{PCa}$ cells that represent the most aggressive circulating $\mathrm{PCa}$ cells using this flow-based device.

\section{Development of prostate metastatic tumors from rolling PC-3 cell implanted mice}

To investigate rolling PCa's metastatic potential, we used an in vivo orthotopic xenograft mouse model. Freshly sorted rolling and floating PC-3 cells were implanted into the anterior prostate of B6 nude mice. Mice were sacrificed 90 days after tumor implantation and several targeted organs were harvested. We found that five of five mice implanted with rolling PC-3 cells showed large prostatic tumors, but strikingly all six mice implanted with floating PC-3 cells did not have any visible tumors within 90 days. Hematoxylin and eosin (H\&E) staining showed normal glandular structures with intact basement membranes of the anterior prostate in all six floating PC-3 cell implanted mice (Figure 3A, first panel). More importantly, we found that four of five mice receiving rolling PC-3 cells developed metastatic tumors in their lymph nodes and lungs as shown in Figure 3A (second and third panel respectively). To confirm that the tumors are indeed derived from human PC-3 cells, we examined the expression of human Pan-cytokeratin (hPCK) by IHC staining and found hPCK positive staining only in the tumor tissues from mice receiving rolling PC-3 cells (Figure 3A, last panel). The development of metastatic tumors in the mice receiving rolling PCa cells strongly supports that the rolling PCa cells represent a subset of population in the circulation that may be responsible for cancer metastasis. The molecular alterations were further examined by the Human Metastasis Tumor Focused Gene Array (SABioscience) by comparing the differentially expressed genes between rolling and floating PCa cells. We found that 26 pro-metastatic genes in PC-3 rolling cells (Figure 3B) and 15 in DU145 rolling cells (Supplementary Figure S3A) were up-regulated in rolling PCa cells. We confirmed five of the common up-regulated genes in both, PC-3 and DU145 cells- IGF-1, MMP3, RORB, SMAD4, and TRPM1, as shown in a Venn diagram (Supplementary Figure S3B) by quantitative PCR using a second primer set (Supplementary Figure S3C). These data suggest that rolling PCa cells contain elevated metastatic related genes that may drive cancer metastasis. 

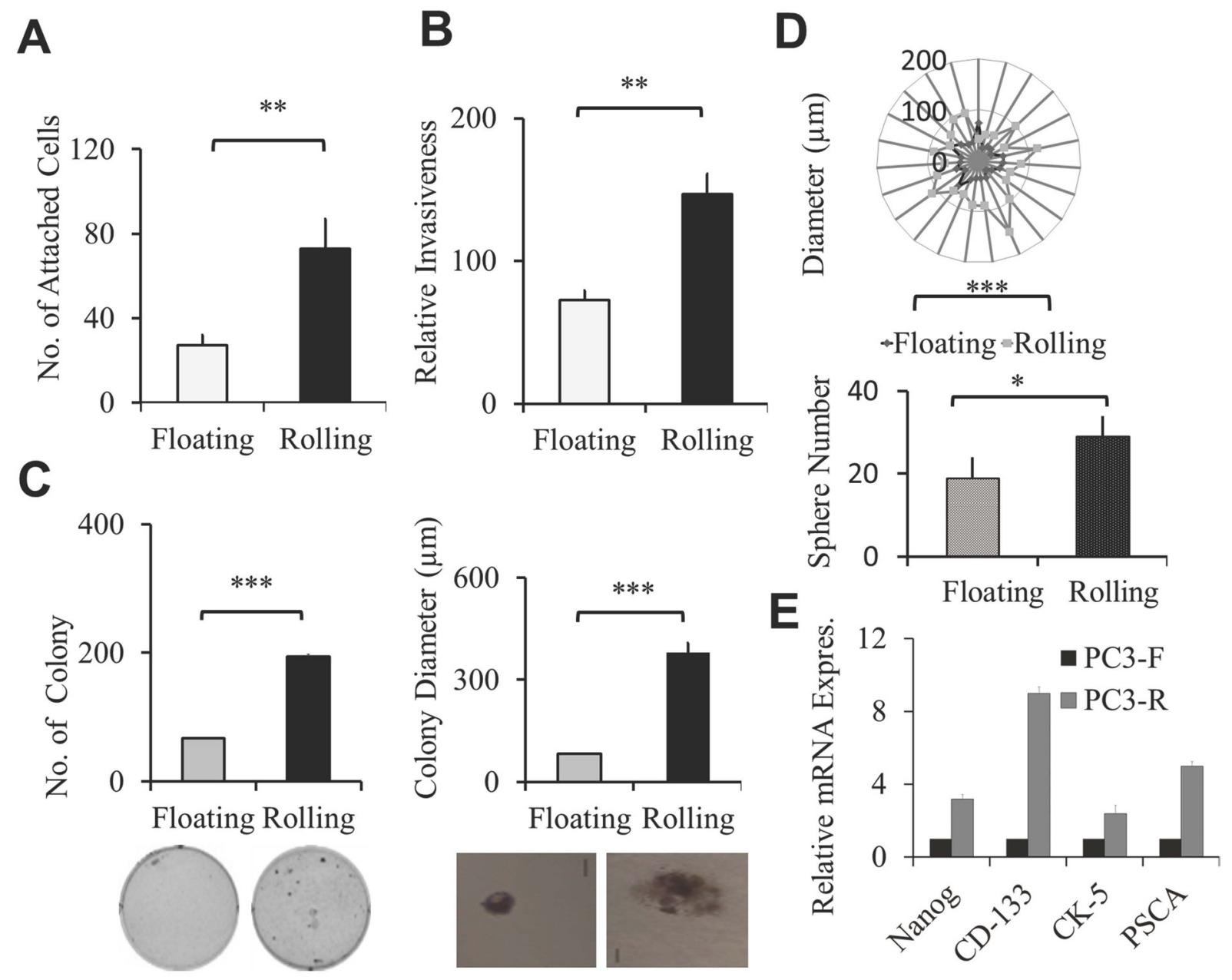

Figure 2: Sorted rolling PCa cells showed higher aggressive cellular cancer phenotype. (A) Static adhesion assay of sorted rolling and floating PC-3 PCa cells on HUVEC cells. Calcein-AM stained rolling and floating PC-3 PCa cells were incubated on the monolayer of HUVEC cells. Adherent cells were counted under fluorescent microscope. Graphs represent the average adherent cell number per well. (B) Invasion assay of sorted rolling and floating PC-3 PCa cells. Bar graph represents relative invasiveness of rolling and floating cells/well in 24 well. (C) Anchorage independent growth by soft agar colony formation assay. Sorted rolling and floating PC-3 cells were seeded on soft agar gel and colony numbers were counted after 21 days. Bar graphs represent average number (left) and average diameter (right) of colony per well of 6 well plate. The pictures represent the size of one colony in 50x view. Scale bar 50 $\mu \mathrm{m}$. (D) 3D sphere forming assay was used to determine the stemness of rolling and floating PC-3 PCa cells. Radar graph shows the diameter of spheres in $\mu \mathrm{m}$, each dot represents one sphere. Bar graph represents the average sphere number per well. (E) Relative mRNA expression of stem cell markers in the sorted rolling and floating PC-3 cells was determined by QPCR. Results represent the average of at least 2 experiments. Error bars indicate SEM. *, $\mathrm{P}<0.05 ; * *, \mathrm{P}<0.01 ; * * *, \mathrm{P}<0.001$.

\section{ESL-1 expression is elevated in rolling PCa cells and correlated with clinical PCa progression}

When PCa cells come in contact with E-selectin coated surfaces, cells tether, roll, and ultimately adhere. The expression/profile of E-selectin ligands on PCa cells might be critical for such interaction that leads to $\mathrm{PCa}$ cells rolling or floating. Thus, we examined the expression levels of E-selectin ligands, CD44, ESL-1 and PSGL-1 on rolling and floating PCa cells. Among the ligands, we found that only ESL-1, but not CD44 or PSGL-1, showed a higher expression level in rolling PC-3 and DU145 cells in mRNA level (Figure 4A). Critically, using flow cytometry we confirmed that rolling $\mathrm{PCa}$ cells express elevated ESL-1 proteins on their surface (Figure 4B and Supplementary Figure S4A). More importantly, this elevated ESL-1 expression was also found in the xenografted prostate tumors derived from mice implanted with rolling PC-3 cells, as compared to prostate of mice receiving floating PC-3 cells by Western blotting analysis (Figure 4C) as well as immunohistochemistry of prostate tissues of floating and rolling PC-3 cell xenografted mice (Figure 4D). These data suggest that ESL-1 represents a novel PCa metastatic marker and its expression level 

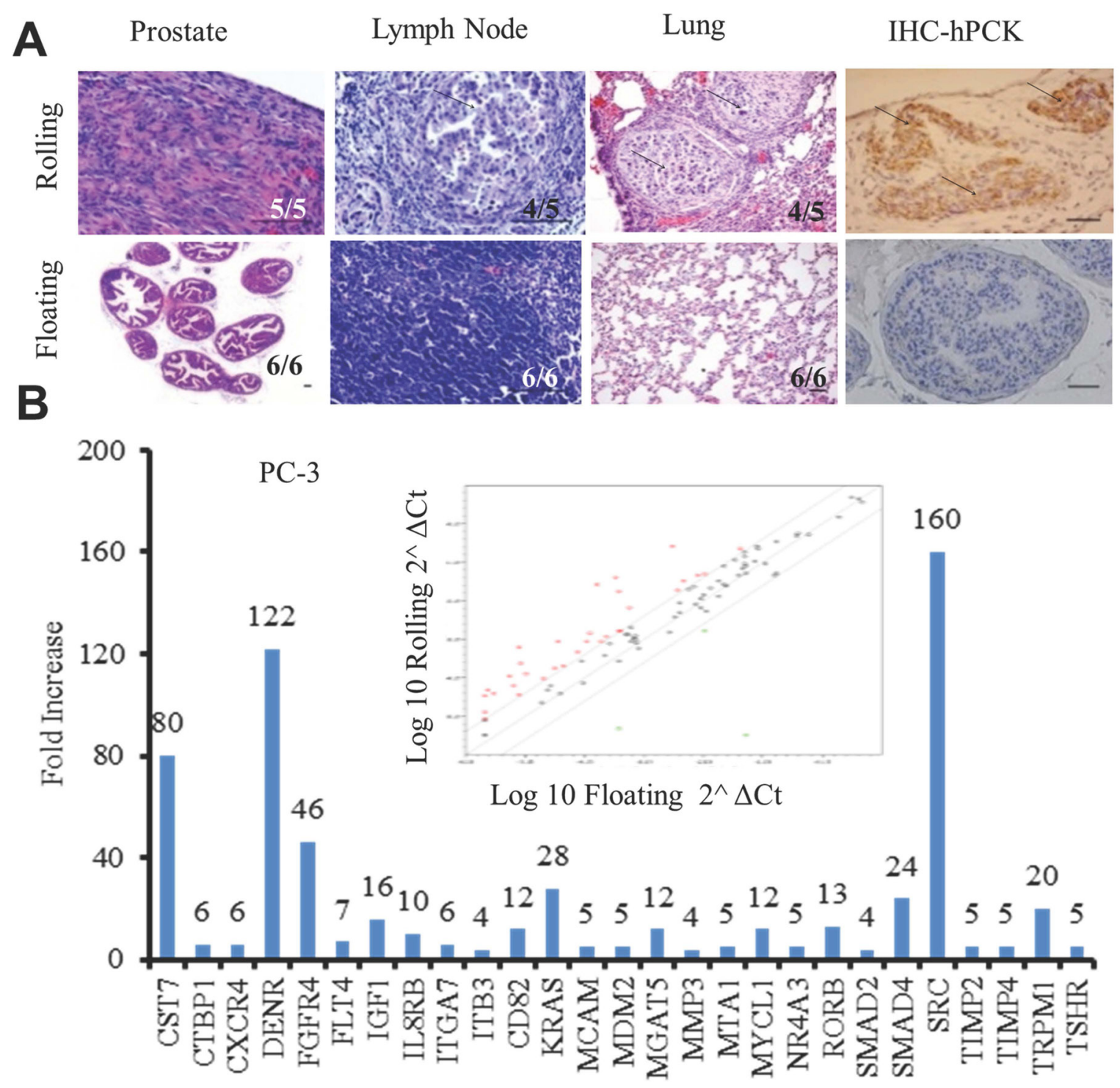

Figure 3: Rolling PCa cells demonstrate higher metastatic behavior in vivo and elevated metastatic related genes. (A) Orthotopic xenograft mouse model was used to determine the metastatic potential of rolling and floating PCa cells in vivo. Mice were sacrificed three months after the implantation, and tissues were collected for histological examination. Histogram of first three columns showing the H\&E staining of the rolling (upper row) and floating (lower row) mouse prostate tumors, lymph node and lung. The $4^{\text {th }}$ column represents the IHC staining of human pan cytokeratin in the rolling (upper) and floating (lower) prostate. Scale bar represents $50 \mu \mathrm{m}$. (B) Human metastatic gene array of 84 metastatic related genes. Scatter plot shows the up- and down-regulated genes (red dots for up and green dots for down) in sorted PC-3 cells and bar graph represents the upregulated metastatic genes ( $>2$ fold) in rolling PC-3 cells, compared with floating.

is correlated with cancer aggressiveness. To confirm this, we examined ESL-1 expression between LNCaP sublines and found that elevated ESL-1 expression in the aggressive LNCaP subline (LNCaP-R) than that in less aggressive LNCaP-P subline (Figure Supplementary S4B). To access the clinical relevance of ESL-1, the ESL-expression in human $\mathrm{PCa}$ samples with different stages of disease were compared through public microarray repositories from NCBI Gene Expression Omnibus (Profile \# GDS2546; metastatic prostate cancer HG-U95B). We compared the expression levels of
ESL-1 in three groups: (1) normal prostate tissue ( $\mathrm{n}=18)$, (2) primary PCa tumors $(\mathrm{n}=63)$ and (3) distant metastasis samples $(n=25)$. We found high levels of ESL-1in metastatic tumors in comparison to normal prostate and primary tumors (Figure 4E), and no significant difference in normal prostate and primary PCa tumors. This elevated level of ESL-1 found in human PCa metastatic samples further supports ESL-1's roles in PCa metastasis; in particular, its status in circulating $\mathrm{PCa}$ cells could be an important functional biomarker for prediction of disease behavior. 


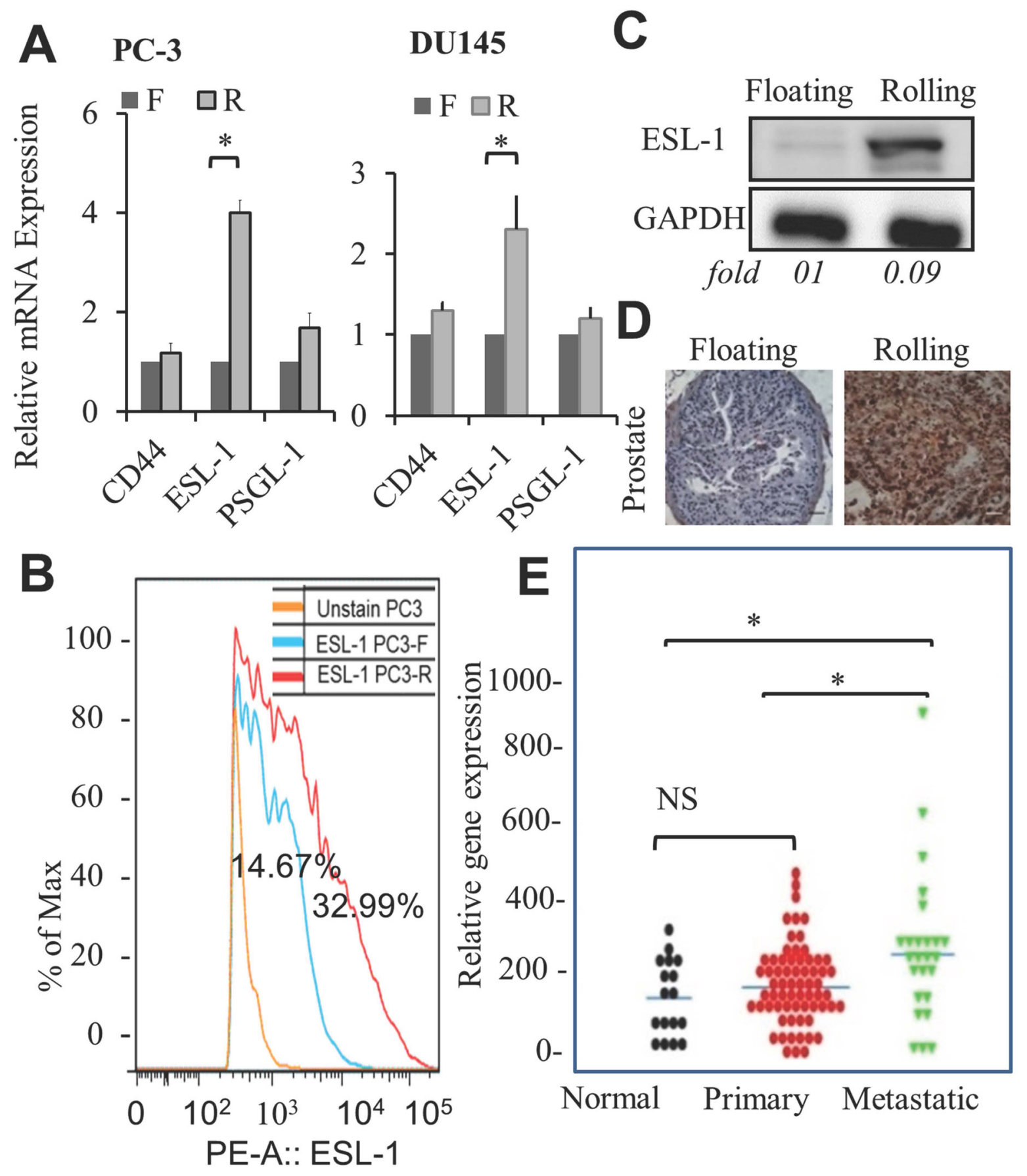

Figure 4: Elevation of ESL-1 in rolling PCa cells, tumors, and human metastatic tissue samples. (A) Relative mRNA expression levels of E-selectin ligands; CD44, ESL-1 and PSGL-1 in sorted PC-3 and DU145 cells were examined by QPCR. Bar graph represents the average of 3 experiments. (B) Flowcytometry data showing surface expression of ESL-1 in sorted rolling and floating PC-3 PCa cells. Blue line represents PC-3 floating cells with surface ESL-1 expression on $14.67 \%$ and red line represents PC-3 rolling cells with surface ESL-1 expression on 32.99\% cells. (C) Western blot analysis of ESL-1 expression in the prostate tissues of xenografted mice implanted with rolling and floating PC-3 cells. (D) ESL-1 expression in same prostate tissues by IHC staining with antibody to human ESL- 1. (E) The gene expression profile of ESL-1 was analyzed through public microarray repositories from NCBI Gene Expression Omnibus (Profile \# GDS2546; Metastatic prostate cancer HG-U95B). Box plots represent expression level of ESL-1 in following 3 categories: normal prostate tissue free of any pathological alteration $(\mathrm{n}=18)$, primary PCa tumors $(\mathrm{n}=63)$ and distant metastasis samples $(\mathrm{n}=25)$. Error bars indicate SEM. *, $\mathrm{P}<0.05$. 


\section{ESL-1 is responsible for PCa cell's rolling and adhesion}

To determine if ESL-1 is a key factor that controls rolling, we knocked down ESL-1 expression in PC-3 and DU145 cells. Two shESL-1 (shESL-1\#4 and shESL-1\#5) and one scramble (scESL-1) stable cell lines were generated. The expression of ESL-1 was determined by QPCR and Western blot, and we showed that both shESL-1\#4 and \#5 ESL-1 clones express significantly lower level of ESL-1 than scESL-1(Figure 5A and B). Those shESL-1 cells were then subjected into in vitro

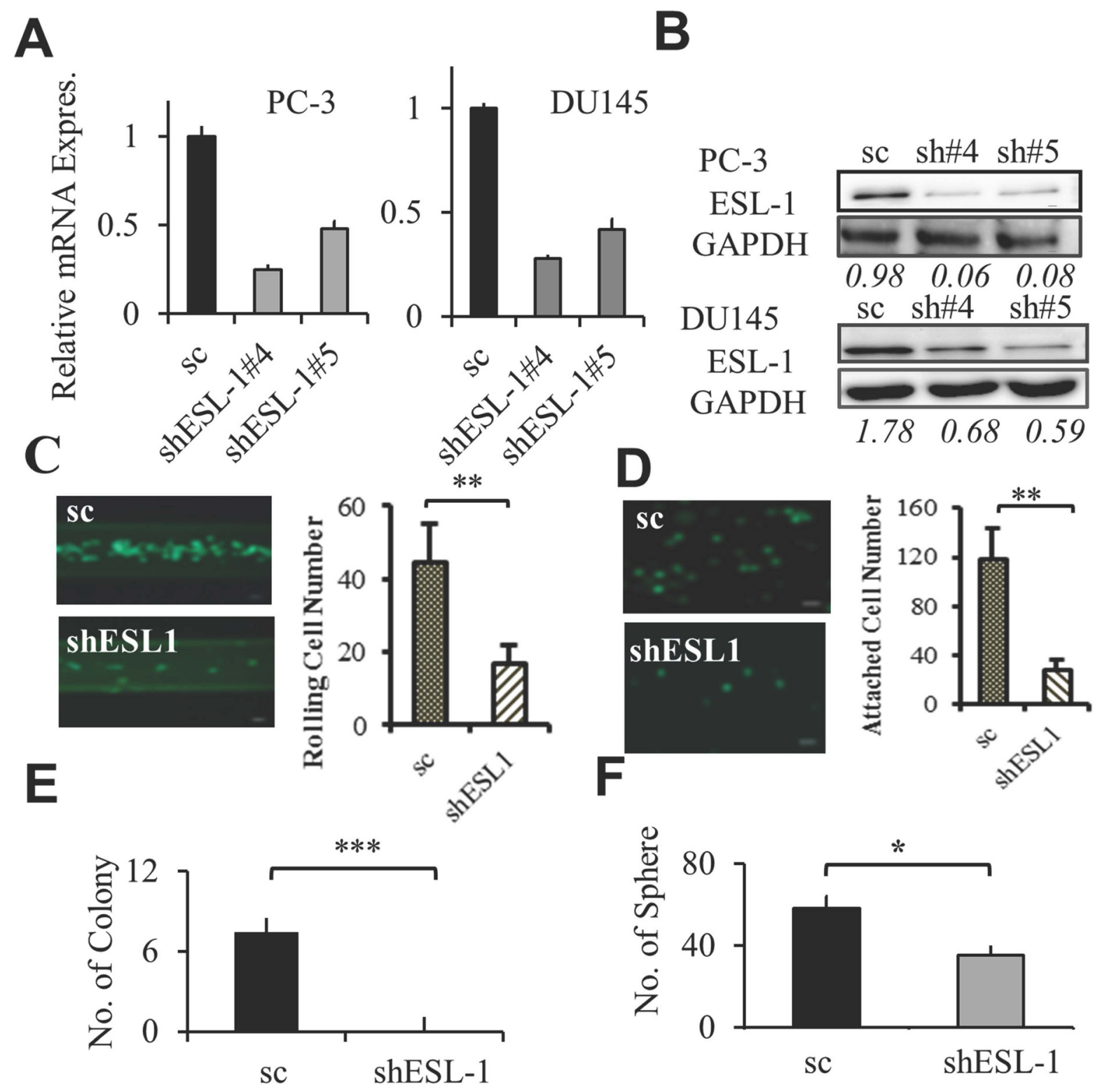

Figure 5: ESL-1 is responsible for PCa rolling and aggressiveness. (A) Expression of ESL-1 in shESL-1 stable clones was determined by Q-PCR. Bar graph represents the fold reduction of ESL-1 expression in shESL-1clones compared to scramble control of PC-3 and DU145 cell lines. (B) Western blot analysis of ESL-1 expression in shESL-1clones compared to scramble control of PC-3 and DU145 cell lines. (C) Determining the rolling cell numbers of the shESL-1and scESL-1 PC-3 PCa cells using the microchannel system under flow of shear stress 1 dyne $/ \mathrm{cm}^{2}$. Bar graph represents the average rolling/adhesion scESL-1 and shESL-1 PC-3 cell number of consecutive 10 frames of videos (100X) of one experiment. A representative picture is shown. Scale bar represents $50 \mu \mathrm{m}$. (D) Comparing the static adhesion between the shESL-1and scESL-1 PC-3 cells. Bar graphs represent the average adherent cell numbers of scESL-1 and shESL-1 PC-3 on HUVEC cells per well and the picture represents 50x view of the attached shESL-1and scESL-1 of PC-3 adherent cells. Scale bar represents $50 \mu \mathrm{m}$. (E) Anchorage independent soft agar colony formation assay with scESL-1and shESL-1 of PC-3 cell line. Colonies were counted after 28 days of culture. Bar graph represents the average colony numbers of three wells in 6 well plate (each were triplicated). (F) 3D sphere forming assay of scESL-1 and shESL-1 of DU145 cell line. Bar graph represents average sphere numbers in three wells of 96 well plates. Results represent the average of at least 3 experiments. Error bars indicate SEM. *, P $<0.05 ; * *, \mathrm{P}<0.01$. 
assays to test their rolling capacity and aggressiveness. As expected, knocking down ESL-1 impairs PCa cells' rolling capacity significantly, where we found a significantly fewer number of shESL-1 PC-3 and shESL-1 DU145 cells can roll and adhere to the coated microtubes under shear stress 1 dyne $/ \mathrm{cm}^{2}$ (Figure 5C and Supplementary Figure S5A). In addition, shESL-1 PCa cells displayed significantly less static adhesion ability than scESL-1cells to the endothelial cells (Figure 5D and Supplementary Figure S5B). Strikingly, the colony formation ability was significantly impaired when ESL-1 was knocked down where shESL-1 PC-3 cells did not develop any significant colony in 28 days of culture (Figure 5E). Interestingly, knocking down ESL-1 also impairs stem cells activity where shESL-1 DU145 cells had reduced number of spheres compared to scESL-1 cells (Figure 5F). In summary, these data support a critical role of ESL-1 not only in controlling cancer cell rolling/adhesion ability, but also in the tumorigenicity, both of which are important for cancer metastasis.

\section{ESL-1 activates RAS-MAP Kinase signaling pathway}

The activation of MAP kinase pathways has been associated with cell migration and invasion [30]. Of particular importance, activation of MAP kinase is associated with cancer metastasis, and elevated levels of phosphorylation of key signals such as ERK1/2 are often found in invasive cancer. To uncover if ESL1 could promote the Ras induced oncogenic pathway, we performed the Western blot and found that knocking down ESL-1 decreased the expression of nRAS, phosphoMEK, phosphoERK1/2 and its downstream transcription factor, cFos (Figure 6A left panel and Supplementary Figure S6). Critically, the elevated expression levels of MAP kinase signal molecules, such as nRAS, pMEK, pERK1/2 and cFOS were found in the prostate tumors derived from the xenografted mice who received high ESL-1 rolling PC-3 cells (Figure 6A. right panel). These data implied that PCa cells express high levels of ESL-1, which may
A

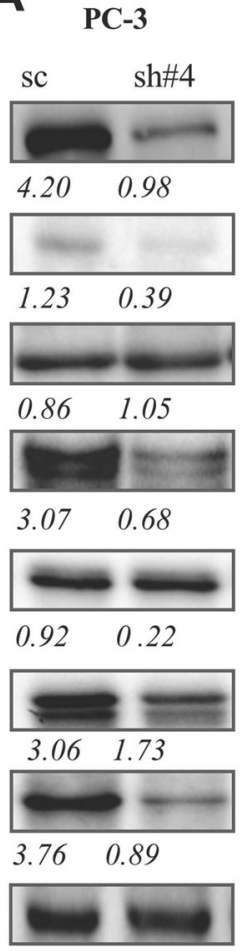

Mice

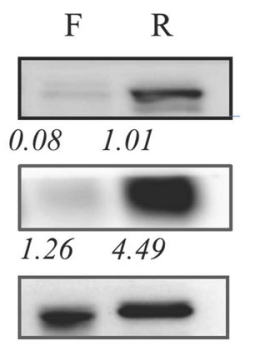

$1.05 \quad 1.14$

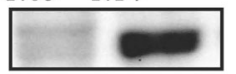

$0.5 \quad 3.23$

Fold

tERK

Fold

pERK

fold

cFOS

fold

GAPDH

B

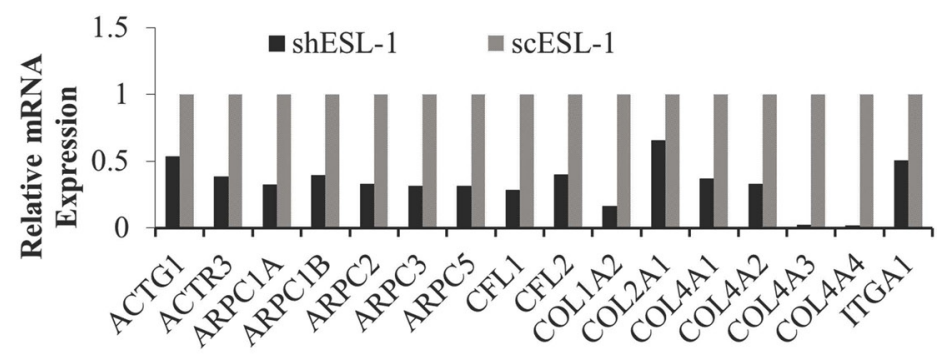

Actin Associated Genes

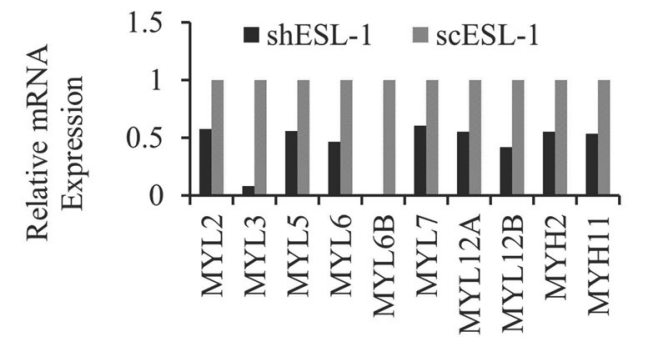

Myosin Associated Genes

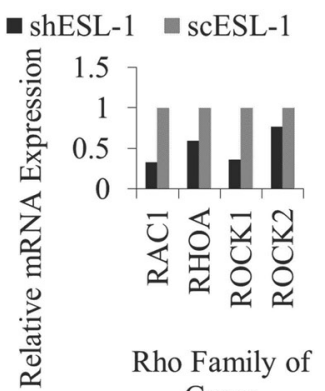

Genes

Figure 6: ESL-1 activates RAS-MAP kinase signaling pathway. (A) Western blot analysis of key molecules of RAS-MAP kinase signaling pathway from cell lysate of shESL-1 and scESL-1 of PC-3 cells (left panel) and prostate tissues from the orthotopic mice receiving rolling and floating PC-3 cells (right panel). They are ESL-1, nRAS, total MEK, phosphoMEK, total ERK1/2, phosphoERK1/2, and its downstream transcription factor, cFOS antibody. GAPDH was used as a housekeeping gene. (B) Integrin-mediated cell adhesion and migration PCR array. Cell adhesion and migration PCR array was performed to compare the cytoskeleton gene expression in scESL-1 and shESL-1 PC-3 cell line. Bar graph represents the relative fold expression of 3 clusters of genes representing Actin, Myosin, and Rho family. 
be responsible for the activation of pMEK and pERK mediated cascade of signaling pathway, consequently enhancing their potential to invade and grow in a second target organ.

To further reveal the ESL-1 downstream target genes in controlling cancer metastasis, we applied the integrin-mediated cell adhesion and migration PCR array (Bio-Rad), because selectins and their ligands orchestrate the cell mobility/adhesion mainly through an integrin dependent mechanism by activating different proteins for cytoskeletal remodeling [31]. As shown in Figure 6B, we found that a number of actin and myosin related genes including Rho family were down-regulated in the shESL-1 cells. Rho GTPases regulate actin polymerization and myosin activity during adhesion dynamics [31]. Our data suggested that ESL-1 is the key factor in circulating PCa cells' rolling/adhesion behavior.

In summary, circulating PCa cells expressing high level of ESL-1 can be stalled and roll on the endothelial cells where E-selectin is expressed. Moreover, we identify ESL-1, as a novel marker for PCa metastasis via controlling cancer cell rolling/adhesion. Overexpression of ESL-1 in prostate cancer cells triggers a signaling kinase cascade, from activation of RAS, MEK, and ERK, consequently inducing the expression transcription factor, and activates a cascade of gene expressions that allows cancer cells to migrate, invade and spread to the secondary organs (Figure 7).

\section{DISCUSSION}

During cancer metastasis, CTCs follow a selectin based migratory pathway that is similar to leukocytes during the inflammatory response [4, 6, 21, 32, 33]. Dimitroff et al have shown that E-selectin ligands, such as PSGL-1, are over-expressed in human prostate bone metastasis tissues [8], suggesting a critical role for E-selectin ligands in cancer metastasis. To date, few studies have reported on the correlation between expression of the E-selectin ligands and prostate cancer bone metastasis [9, 12, 18, 34]. In this study, we identified ESL-1 as the key factor that controls PCa cells' rolling capacity under the flow, and knocking down ESL-1 impairs PCa cell rolling and mobility, and disrupts anchorage independent growth. This is the first study that demonstrates the functional roles of ESL-1 in controlling PCa rolling/adhesion and metastasis. ESL-1 is a $\mathrm{Ca}^{2+}$ dependent inducible cell adhesion glycoprotein, responsible for converting the initial tethering into slow rolling in myeloid cells [35]. It is also believed to be a variant of the receptor of fibroblast growth factor (FGF) $[36,37]$, which may demonstrates the interesting relationship between the high expression of ESL-1 with PCa growth rate, as FGF is known to be one of the growth factors in cancer progression [38].

The ESL-1 mediated pathways that control cancer cell adhesion/rolling and metastasis are largely unknown.

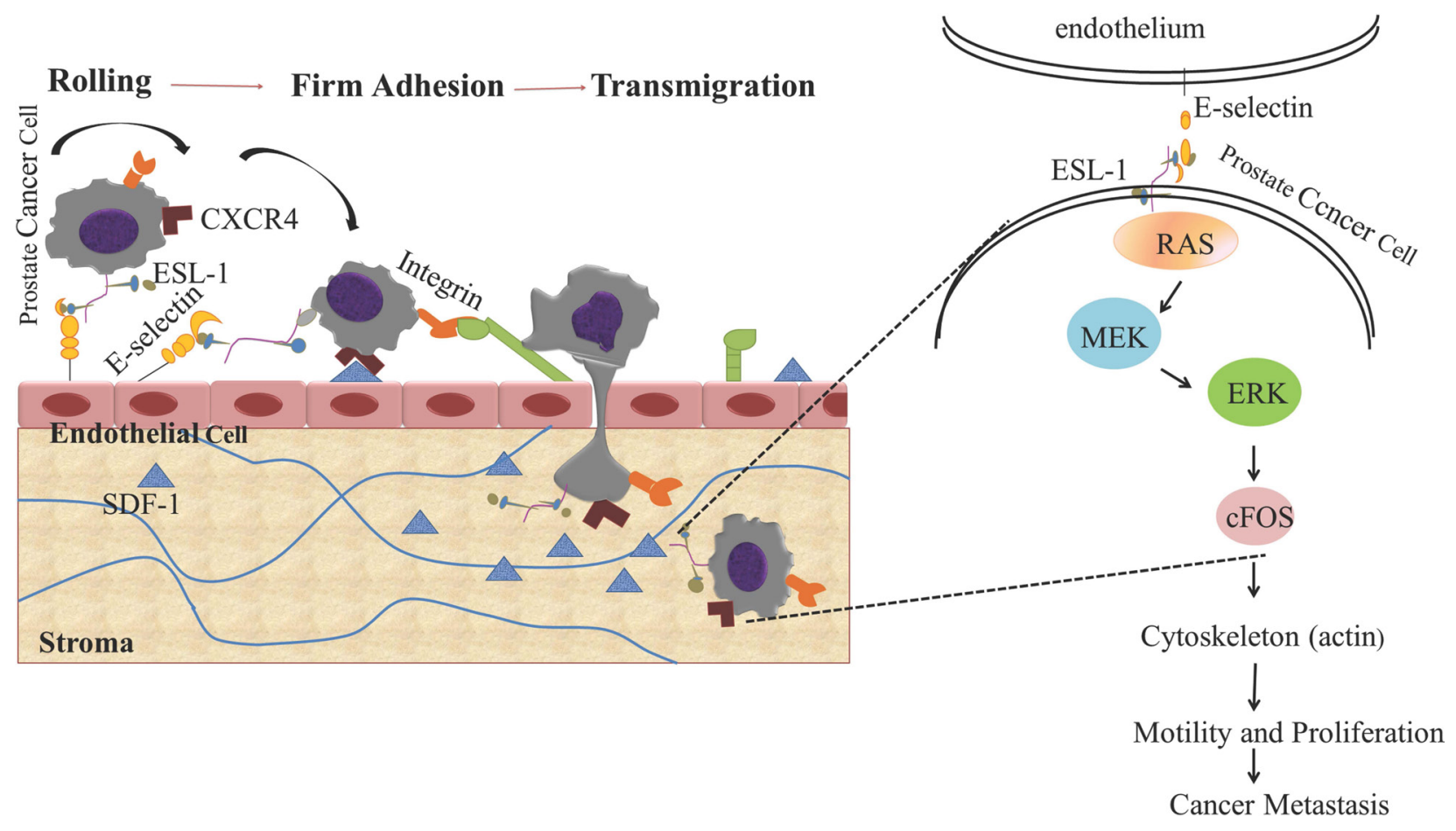

Figure 7: Schematic diagram of the oncogenic signaling pathways induced by E-selectin/ESL-1- mediated PCa rolling and adhesion on endothelial cell surface. PCa cells express high level of E-selectin ligand, ESL-1, which mediates the rolling/ adhesion of PCa cells on endothelial surface through the RAS-ERK- MAP kinase pathway. 
In this report, we showed that overexpression of ESL-1 activates Ras-MAP kinase cascade in PCa cells, then triggers the expression of downstream targets, like integrin mediated cytoskeleton system (action and myosin polymerization) and the Rho family of proteins [31]. The ERK signaling pathway is known to control diverse cellular processes, and was found to be often up-regulated in human tumors. ERK signal has been found to be important for the osteomimetic properties of the bone metastasis of prostate cancer cell lines [39], and expression of the key signal factors such as MEK5 is found to be overexpressed in PCa metastatic tissues [40] and can promote PCa cell mobility. Moreover, we also identified ESL-1 downstream targeted genes that are involved in the cytoskeleton movement. All of these proteins are required for cell mobility, cell migration, and cell adhesion and all those cellular behaviors are essential for cancer progression during distant metastasis. In summary, our study reported a novel pathway that controls cell rolling and adhesion, from ESL-1 to RASERK, then to cytoskeleton proteins. Targeting ESL-1 and/ or its regulatory signal molecules, and/or downstream oncogenic proteins may provide a novel therapeutic strategy, especially for those patients who have a high ESL-1 expression in CTCs.

Our findings on ESL-1's oncogenic properties and its elevated expression profile in clinical metastases are clinically significant. First, ESL-1 can be used as a novel functional biomarker for CTCs that can be used to predict patients' outcomes, i.e., to include not only patients' CTCs numbers, but also the expression level of ESL-1 to determine CTCs" "bioactivity". More sensitive assays, such as an immunofluorescence assay, and an in-depth large scale clinical study are required to further establish the correlation of ESL-1 in CTCs with patients' outcomes. Second, this identified oncogenic pathway(s) induced by ESL-1 can serve as a principle for developing targeted therapy for those patients who have higher levels of ESL1 in their CTCs.

Metastasis is the most dreaded stage of cancer and a major cause of death from prostate cancer. Therefore, understanding the $\mathrm{PCa}$ metastatic process, and identifying the essential molecular events during metastases are important tasks. Our study provides a model system to isolate and characterize a small rolling population of CTCs, and identifies a key molecule, ESL1, for its roles in CTCs rolling and cancer metastasis. Further clinical evaluation of the expression levels of ESL-1 in patients and corresponding clinical outcomes are highly anticipated, and better therapeutics designed to block CTCs rolling and adhesion require further investigation.

\section{MATERIALS AND METHODS}

\section{Cell Lines, molecules, and antibodies}

BPH-1 series: PHEC $_{\mathrm{T}}$ and $\mathrm{PHEC}_{\mathrm{M}}$ human prostate epithelial BPH-1 series of cells were provided by Dr. William Ricke. BPH-1 cells undergo malignant transformation when they are recombined with mouse urogenical mesenchymal cells (mUGM) under the kidney capsule. PHEC $_{\mathrm{T}}$ was derived from non-metastatic primary tumor and $\mathrm{PHEC}_{\mathrm{M}}$ was derived from lymph node metastasis [24]. LNCaP-P and LNCaP-R, were established and CWR22R-1 and CWR22R-2 were tested in our laboratory where LNCaP-R and CWR22R-2 cells are resistant to chemotherapy drugs such as vitamin $\mathrm{D}$ and exhibit more aggressive behavior as compared to their parental line (LNCaP-P and CWR22R-1) [25, 26]. PC-3 and DU145 cell lines were obtained from American Type Culture Collection (ATCC). Primary human umbilical vein endothelial cells (HUVEC) were a generous gift from the Aab Cardiovascular Research Institute of the University of Rochester. PHEC ${ }_{\mathrm{T}}$ and $\mathrm{PHEC}_{\mathrm{M}}$, LNCaP-P, LNCaP-R, CWR22R-1, CWR22R-2, PC-3, and DU145 cell lines were cultured with RPMI-1640 medium (GIBCO) containing 10\% fetal bovine serum and 1\% streptomycin/penicillin. Primary Human Umbilical Vein Endothelial Cells (HUVEC) were maintained in Medium 200 (Gibco, Carlsbad, CA), containing 1x low LSGS and $2 \%$ heat-inactivated fetal bovine serum [41]. Recombinant human E-selectin and SDF-1 $\beta$ were purchased from R\&D systems.

\section{Preparation of microtube for cell rolling and capture}

The inner surface of the blood vessel-compatible Micro-Renathane tube (MRE 025, inside diameter $300 \mu \mathrm{m}$, length $50 \mathrm{~cm}$, Braintree Scientific Inc.) was coated with recombinant human E-selectin/Fc chimera (rhE/Fc, R\&D Systems), and SDF-1 $\beta$ (R\&D Systems) at $40 \mu \mathrm{g} / \mathrm{ml}$ and $10 \mu \mathrm{g} / \mathrm{ml}$ concentrations respectively in Dulbecco's Phosphate Buffered saline (DPBS, GIBCO) by incubating two hours at room temperature. Then, the microtubes were gently and slowly washed with calciumenriched Hanks balanced salt solution $\left(\mathrm{HBSS}^{+}, \mathrm{pH}\right.$ 7.4, $2 \mathrm{mM} \mathrm{Ca}^{++}, \mathrm{GIBCO}$ ) [23] and after 15 minutes were ready for rolling assay.

\section{PCa cell rolling on microtube}

PCa cells were detached from the culture dish surface by cell dissociation buffer (GIBCO) for 
10 minutes, and washed with cell culture medium. Cells were suspended in $\mathrm{Ca}^{++} \mathrm{HBSS}$ buffer for 15 to 30 minutes on ice. Cell numbers and viability were determined, and cell density was controlled at $1-2 \times 10^{5}$ cells $/ \mathrm{ml}$. MRE tubes were positioned on the stage of an Olympus IX-81 microscope coupled to a Hitachi CCD camera KP-M1AN (Hitachi, Tokyo, Japan) for direct visualization of the cells in the lumen [23]. PCa cells were infused through the tubes at $1 \mathrm{dyne} / \mathrm{cm}^{2}$ wall shear stress using a syringe pump (New Era) (Supplementary Figure S1). The images were captured using StreamPix and data were analyzed.

\section{Determining rolling cell number and rolling velocity}

Rolling PCa cells were defined as cells translating at $<50 \%$ of the calculated hydrodynamic free stream velocity and cells that remained stationary for more than 10 seconds or rolled less than four cell diameters were considered as rolling cell [42]. The rolling cell number was determined as the average cells captured from 10 consecutive frames of the film captured at 200X. Image J and Excel software were used to measure the change in position of the cell center in a given period of time to calculate the rolling velocity. The time and distance of travel of 10 random rolling cells from each one minute of film was calculated. Rolling velocity was calculated [23].

\section{Sorting PCa cells}

To sort $\mathrm{PCa}$ cells into rolling and floating subpopulations, PC-3 and DU145 cells were prepared [43] and infused through coated MRE tubes at a physiological capillary shear stress of $1 \mathrm{dyne} / \mathrm{cm}^{2}$ [44]. After 30 minutes of infusion, each tube was infused with $\mathrm{Ca}^{++} \mathrm{HBSS}$ buffer to wash out all of the unbound cells. The unattached (named as "floating") cells were collected at the other end of the tube. Cells adhering to the tube (named as "rolling") were collected by using a high shear with calcium free DPBS. Cell number count and viability of the collected cells were analyzed using a Vi-Cell XR Cell Viability AnalyZer (Beckman Coulter) and $>85 \%$ viable cells were used for experiments. Also to reduce the heterogeneity of the cells during cell culture, only freshly sorted cells were used in all of the experiments.

\section{Static adhesion assay}

HUVEC cells were grown as a monolayer in a 6-well plate to obtain confluence up to $90 \%$. Freshly sorted "floating" and "rolling" PCa cells were labeled with GFP by incubating with $1 \mu \mathrm{M}$ Calcein-AM fluorescent dye (Sigma) for 30 minutes at $37^{\circ} \mathrm{C}$ and washed with PBS. Floating and rolling Calcein-AM labeled PCa cells were seeded on the monolayer of HUVEC cells $\left(5 \times 10^{3} /\right.$ well $)$ and incubated for 30 minutes at $37^{\circ} \mathrm{C}$ with $5 \% \mathrm{CO}_{2}$. Unbound
PCa cells were washed out with PBS. Adherent cells were counted and photographs were taken using a Zeiss Axio A1 microscope coupled with Zenoptik camera measuring the intensity of fluorescent at 494/517 nm (Abs/Em) [23]. For shESL-1 and scESL-1 PCa cells, no Calcein-AM labeling was required since both cells express GFP.

\section{Sphere forming assay}

Single cell suspension was seeded at a density of 100 cells/well in 96 well plates on the top of a thin layer of matrigel (BD Bioscience) [45]. RPMI cell culture media with $10 \%$ FBS and $4 \%$ matrigel was added on the top every other day. Cells were cultured up to 8 days. Colonies were counted and photographs were taken using a Zeiss Axio A1 microscope coupled with Zenoptik camera.

\section{Colony forming assay}

In an anchorage-independent colony forming assay, sorted PCa cells were suspended at a density of $10^{3}$ cells/ well in $0.4 \%$ low melting point agarose in RPMI cell culture media, and plated on top of $2 \mathrm{ml}$ underlayer of $0.8 \%$ agarose in the same medium, in 6-well culture plates. Medium was refreshed twice per week for three weeks, stained with p-iodonitrotetrazolium violet and colonies containing more than 50 cells were counted and photographed with a Zeiss Axio A1 microscope coupled with Zenoptik camera. Diameter was measured from the photograph using Image J software [26, 46].

\section{Flow cytometry}

Expression of ESL-1 was analyzed by Flow cytometry (BD FACS Canto II) following standard protocol [47] and was analyze using FlowJo software. Anti ESL-1 antibody (ab58826 from ABCam) and PE labeled secondary antibody was used from Miltenyi Biotech following the manufacturer's protocol. To detect the total ESL-1, cells were permeabilized with $0.1 \%$ PBSTween for 20 minutes. Cells were prepared following the manufacturer's instruction.

\section{Human metastatic and adhesion/migration gene array}

To compare the gene profile alteration on the two sorted subpopulations of PCa cells, tumor metastasis focus using RT2 Profiler PCR Array of Human Tumor Metastasis (SABioscience) kit was used to do metastatic gene array to determine and compare up and down regulated metastatic gene among sorted "floating" and "rolling" PCa cell population. In addition, we also applied Integrin Mediated Cell Adhesion and Migration H96 predesigned 96 well array panel (BioRad). 


\section{Orthotopic xenograft}

$1 \times 10^{6}$ sorted, viable (>85\%), PC-3 floating and rolling cells suspended in BD Matrigel were implanted to the anterior prostate of 10 week old nude mice ( 6 for floating and 5 for rolling) [22]. The mice were euthanized after 90 days, the prostate, adjacent lymph nodes, liver, and lungs were collected for histological analysis, and targeted proteins expression was measured by Western blotting analysis. Photographs were taken using a Cannon digital camera and histological photographs were taken using a Zeiss Axio A1 microscope coupled with Zenoptik camera and Leica DM5000 B microscope coupled with Optronics MacroFire digital camera.

\section{Immunohistochemistry (IHC) staining}

IHC staining was performed following the standard protocol [48]. The sections were stained with the primary antibody before being analyzed with the commercial kit. The sections were counterstained with Hematoxylin (Ricca Chemical Company Catalogue No. 3530-16) and rinsed with tap water. Sections were observed and the micrographs were pictured using Leica DM5000 B microscope coupled with Optronics MacroFire digital camera. Antibodies for IHC: Cocktail of Human Pancytokeratin was used to identify human cytokeratin in the human PC-3 cell implanted metastatic cancers. Dilution was 1:200. Mixture of 2 antibodies was used: BD Bioscience, antihuman-cytokeratin,CAM 5.2 and Dako, monoclonal mouse anti-human cytokeratin, \#M3515, clone: AE1/AE3. For staining ESL-1 anti ESL-1 antibody (Abcam, Cat \# ab103439) was used in prostate tissues.

\section{Establishment of shESL-1 PCa cells}

ESL-1 shRNA and universal scramble control plasmids were constructed in lentiviral pGIPZ vector (Open Biosystems) following the company protocol. To establish the stable cells we transfected pGIPZ -scramble or ESL-1 shRNA (pGIPZ -ESL-1 shRNA) with pGIPZ - psPAX2 (virus-packaging plasmid), and $\mathrm{pMD}_{2} \mathrm{G}$ (envelope plasmid) into 293 cells using Lipofectamine 2000 (Invitrogen). The viral containing supernatants were collected 48-72 hours after transfection, infected into prostate cancer cells (PC-3 and DU145) and selected for stable gene insertion under $2 \mu \mathrm{g} / \mathrm{ml}$ puromycin.

\section{Statistical analysis}

A paired t- test was used for statistically analyzing the data, and s5 significance is defined as $\mathrm{P}<0.05$. text)

(Details materials and methods in supplementary

\section{ACKNOWLEDGEMENTS}

The authors thank Dr. William Ricke at University of Wisconsin Madison, for supplying the BPH-1 cell line series. Dr. Martha Blair, Department of Pharmacology and Physiology University of Rochester, Dr. Hiroshi Miyamoto at Johns Hopkins University, and Dr. Shengqiang Yu at Shanghai Jiao Tong University, China for the technical support. This study was funded by the Department of Defense Prostate Cancer Research Program Predoctoral Training Award (\# W81XWH-11-1-0429) to Sayeda Yasmin-Karim.

\section{Conflict of interest}

Authors declare no conflict of interest.

\section{REFERENCES}

1. Lange PH, Vessella RL. Mechanisms, hypotheses and questions regarding prostate cancer micrometastases to bone. Cancer metastasis reviews. 1998; 17:331-336.

2. Aguirre-Ghiso JA. Models, mechanisms and clinical evidence for cancer dormancy. Nature reviews Cancer. 2007; 7:834-846.

3. Jacobs PP, Sackstein R. CD44 and HCELL: preventing hematogenous metastasis at step 1. FEBS letters. 2011; 585:3148-3158.

4. Bonnomet A, Brysse A, Tachsidis A, Waltham M, Thompson EW, Polette M, Gilles C. Epithelial-tomesenchymal transitions and circulating tumor cells. Journal of mammary gland biology and neoplasia. 2010; 15:261-273.

5. He H, Yang $\mathrm{X}$, Davidson $\mathrm{AJ}, \mathrm{Wu} \mathrm{D}$, Marshall FF, Chung LW, Zhau HE, Wang R. Progressive epithelial to mesenchymal transitions in ARCaP E prostate cancer cells during xenograft tumor formation and metastasis. The Prostate. 2010; 70:518-528.

6. Gajula RP, Chettiar ST, Williams RD, Thiygarajan S, Kato Y, Aziz K, Wang R, Gandhi N, Wild AT, Vesuna F, Ma J, Salih T, Cades J, Fertig E, Biswal S, Burns TF, et al. The Twist Box Domain is Required for Twist1-induced Prostate Cancer Metastasis. Mol Cancer Res. 2013.

7. Carvalho FL, Simons BW, Antonarakis ES, Rasheed Z, Douglas N, Villegas D, Matsui W, Berman DM. Tumorigenic potential of circulating prostate tumor cells. Oncotarget. 2013; 4:413-421.

8. Dimitroff CJ, Lechpammer M, Long-Woodward D, Kutok JL. Rolling of human bone-metastatic prostate tumor cells on human bone marrow endothelium under shear flow is mediated by E-selectin. Cancer research. 2004; 64:5261-5269.

9. Barthel SR, Gavino JD, Wiese GK, Jaynes JM, Siddiqui J, Dimitroff CJ. Analysis of glycosyltransferase expression in metastatic prostate cancer cells capable of rolling activity 
on microvascular endothelial (E)-selectin. Glycobiology. 2008; 18:806-817.

10. Barthel SR, Hays DL, Yazawa EM, Opperman M, Walley KC, Nimrichter L, Burdick MM, Gillard BM, Moser MT, Pantel K, Foster BA, Pienta KJ, Dimitroff CJ. Definition of molecular determinants of prostate cancer cell bone extravasation. Cancer research. 2013; 73:942-952.

11. Sreeramkumar V, Leiva M, Stadtmann A, Pitaval C, OrtegaRodriguez I, Wild MK, Lee B, Zarbock A, Hidalgo A. Coordinated and unique functions of the E-selectin ligand ESL-1 during inflammatory and hematopoietic recruitment in mice. Blood. 2013.

12. Dimitroff CJ, Descheny L, Trujillo N, Kim R, Nguyen V, Huang W, Pienta KJ, Kutok JL, Rubin MA. Identification of leukocyte E-selectin ligands, P-selectin glycoprotein ligand-1 and E-selectin ligand-1, on human metastatic prostate tumor cells. Cancer research. 2005; 65:5750-5760.

13. Gout S, Morin C, Houle F, Huot J. Death receptor-3, a new E-Selectin counter-receptor that confers migration and survival advantages to colon carcinoma cells by triggering p38 and ERK MAPK activation. Cancer research. 2006; 66:9117-9124.

14. Tozeren A, Kleinman HK, Grant DS, Morales D, Mercurio AM, Byers SW. E-selectin-mediated dynamic interactions of breast- and colon-cancer cells with endothelial-cell monolayers. International journal of cancer Journal international du cancer. 1995; 60:426-431.

15. Fukuda MN, Ohyama C, Lowitz K, Matsuo O, Pasqualini R, Ruoslahti E, Fukuda M. A peptide mimic of E-selectin ligand inhibits sialyl Lewis X-dependent lung colonization of tumor cells. Cancer research. 2000; 60:450-456.

16. Dallas MR, Chen SH, Streppel MM, Sharma S, Maitra A, Konstantopoulos K. Sialofucosylated podocalyxin is a functional E- and L-selectin ligand expressed by metastatic pancreatic cancer cells. American journal of physiology Cell physiology. 2012; 303:C616-624.

17. Li J, Guillebon AD, Hsu JW, Barthel SR, Dimitroff CJ, Lee YF, King MR. Human fucosyltransferase 6 enables prostate cancer metastasis to bone. Br J Cancer. 2013.

18. Barthel SR, Gavino JD, Descheny L, Dimitroff CJ. Targeting selectins and selectin ligands in inflammation and cancer. Expert opinion on therapeutic targets. 2007; 11:1473-1491.

19. Laubli H, Borsig L. Selectins promote tumor metastasis. Seminars in cancer biology. 2010; 20:169-177.

20. Borsig L, Vlodavsky I, Ishai-Michaeli R, Torri G, Vismara E. Sulfated hexasaccharides attenuate metastasis by inhibition of P-selectin and heparanase. Neoplasia. 2011; 13:445-452.

21. Ley K, Laudanna C, Cybulsky MI, Nourshargh S. Getting to the site of inflammation: the leukocyte adhesion cascade updated. Nature reviews Immunology. 2007; 7:678-689.

22. Taichman RS, Cooper C, Keller ET, Pienta KJ, Taichman NS, McCauley LK. Use of the stromal cell- derived factor-1/CXCR4 pathway in prostate cancer metastasis to bone. Cancer research. 2002; 62:1832-1837.

23. Hsu JW, Yasmin-Karim S, King MR, Wojciechowski JC, Mickelsen D, Blair ML, Ting HJ, Ma WL, Lee YF. Suppression of prostate cancer cell rolling and adhesion to endothelium by 1alpha,25-dihydroxyvitamin D3. The American journal of pathology. 2011; 178:872-880.

24. Lapek JD, Jr., McGrath JL, Ricke WA, Friedman AE. LC/ LC-MS/MS of an innovative prostate human epithelial cancer (PHEC) in vitro model system. Journal of chromatography B, Analytical technologies in the biomedical and life sciences. 2012; 893-894:34-42.

25. Bao BY, Ting HJ, Hsu JW, Yasmin-Karim S, Messing E, Lee YF. Down-regulation of NF-kappaB signals is involved in loss of 1alpha, 25-dihydroxyvitamin D3 responsiveness. J Steroid Biochem Mol Biol. 2010; 120:11-21.

26. Ting HJ, Bao BY, Reeder JE, Messing EM, Lee YF. Increased expression of corepressors in aggressive androgen-independent prostate cancer cells results in loss of 1alpha,25-dihydroxyvitamin D3 responsiveness. Mol Cancer Res. 2007; 5:967-980.

27. Brennen WN, Chen S, Denmeade SR, Isaacs JT. Quantification of Mesenchymal Stem Cells (MSCs) at sites of human prostate cancer. Oncotarget. 2013; 4:106-117.

28. Amini S, Fathi F, Mobalegi J, Sofimajidpour H, Ghadimi T. The expressions of stem cell markers: Oct4, Nanog, Sox2, nucleostemin, Bmi, Zfx, Tcl1, Tbx3, Dppa4, and Esrrb in bladder, colon, and prostate cancer, and certain cancer cell lines. Anatomy \& cell biology. 2014; 47:1-11.

29. Reiter RE, Gu Z, Watabe T, Thomas G, Szigeti K, Davis E, Wahl M, Nisitani S, Yamashiro J, Le Beau MM, Loda M, Witte ON. Prostate stem cell antigen: a cell surface marker overexpressed in prostate cancer. Proceedings of the National Academy of Sciences of the United States of America. 1998; 95:1735-1740.

30. Guo G, Yao W, Zhang Q, Bo Y. Oleanolic acid suppresses migration and invasion of malignant glioma cells by inactivating MAPK/ERK signaling pathway. PloS one. 2013; 8:e72079.

31. Parsons JT, Horwitz AR, Schwartz MA. Cell adhesion: integrating cytoskeletal dynamics and cellular tension. Nature reviews Molecular cell biology. 2010; 11: 633-643.

32. Sackstein R. The bone marrow is akin to skin: HCELL and the biology of hematopoietic stem cell homing. The journal of investigative dermatology Symposium proceedings / the Society for Investigative Dermatology, Inc [and] European Society for Dermatological Research. 2004; 9:215-223.

33. Julien S, Ivetic A, Grigoriadis A, QiZe D, Burford B, Sproviero D, Picco G, Gillett C, Papp SL, Schaffer L, Tutt A, Taylor-Papadimitriou J, Pinder SE, Burchell JM. Selectin ligand sialyl-Lewis $\mathrm{x}$ antigen drives metastasis of hormone-dependent breast cancers. Cancer research. 2011; 71:7683-7693. 
34. Gout S, Tremblay PL, Huot J. Selectins and selectin ligands in extravasation of cancer cells and organ selectivity of metastasis. Clinical \& experimental metastasis. 2008; 25:335-344.

35. Hidalgo A, Peired AJ, Wild MK, Vestweber D, Frenette PS. Complete identification of E-selectin ligands on neutrophils reveals distinct functions of PSGL-1, ESL-1, and CD44. Immunity. 2007; 26:477-489.

36. Steegmaier $\mathrm{M}$, Levinovitz A, Isenmann $\mathrm{S}$, Borges $\mathrm{E}$, Lenter M, Kocher HP, Kleuser B, Vestweber D. The E-selectin-ligand ESL-1 is a variant of a receptor for fibroblast growth factor. Nature. 1995; 373:615-620.

37. Mourelatos Z, Gonatas JO, Nycum LM, Gonatas NK, Biegel JA. Assignment of the GLG1 gene for MGF-160, a fibroblast growth factor and E-selectin binding membrane sialoglycoprotein of the Golgi apparatus, to chromosome 16 q22-q23 by fluorescence in situ hybridization. Genomics. 1995; 28:354-355.

38. Turner N, Grose R. Fibroblast growth factor signalling: from development to cancer. Nature reviews Cancer. 2010; 10:116-129.

39. Zayzafoon M, Abdulkadir SA, McDonald JM. Notch signaling and ERK activation are important for the osteomimetic properties of prostate cancer bone metastatic cell lines. The Journal of biological chemistry. 2004; 279:3662-3670.

40. Mehta PB, Jenkins BL, McCarthy L, Thilak L, Robson CN, Neal DE, Leung HY. MEK5 overexpression is associated with metastatic prostate cancer, and stimulates proliferation, MMP-9 expression and invasion. Oncogene. 2003; 22:1381-1389.

41. Hsu WY, Chao YW, Tsai YL, Lien CC, Chang CF, Deng MC, Ho LT, Kwok CF, Juan CC. Resistin induces monocyte-endothelial cell adhesion by increasing
ICAM-1 and VCAM-1 expression in endothelial cells via p38MAPK-dependent pathway. Journal of cellular physiology. 2011; 226:2181-2188.

42. King MR, Hammer DA. Multiparticle adhesive dynamics: hydrodynamic recruitment of rolling leukocytes. Proceedings of the National Academy of Sciences of the United States of America. 2001; 98:14919-14924.

43. Rana K, Liesveld JL, King MR. Delivery of apoptotic signal to rolling cancer cells: a novel biomimetic technique using immobilized TRAIL and E-selectin. Biotechnology and bioengineering. 2009; 102:1692-1702.

44. Giavazzi R, Foppolo M, Dossi R, Remuzzi A. Rolling and adhesion of human tumor cells on vascular endothelium under physiological flow conditions. The Journal of clinical investigation. 1993; 92:3038-3044.

45. Fan X, Liu S, Su F, Pan Q, Lin T. Effective enrichment of prostate cancer stem cells from spheres in a suspension culture system. Urologic oncology. 2012; 30:314-318.

46. Ting HJ, Messing J, Yasmin-Karim S, Lee YF. Identification of microRNA-98 as a therapeutic target inhibiting prostate cancer growth and a biomarker induced by vitamin D. The Journal of biological chemistry. 2013; 288:1-9.

47. Demaret J, Walencik A, Jacob MC, Timsit JF, Venet F, Lepape A, Monneret G. Inter-laboratory assessment of flow cytometric monocyte HLA-DR expression in clinical samples. Cytometry Part B, Clinical cytometry. 2013; 84:59-62.

48. Cai Y, Lee YF, Li G, Liu S, Bao BY, Huang J, Hsu CL, Chang C. A new prostate cancer therapeutic approach: combination of androgen ablation with COX-2 inhibitor. International journal of cancer Journal international du cancer. 2008; 123:195-201. 